\title{
The Effect of Sungold Kiwifruit (Actinidia Chinensis var. Chinensis) on Gut Health Function: A Randomized Cross-Over Clinical Trial ${ }^{\dagger}$
}

\author{
Alison Wallace 1,*, Sarah Eady 1, Christine Butts ${ }^{2}$, Duncan Hedderley ${ }^{2}$, Lynley Drummond ${ }^{3}$, \\ Juliet Ansell ${ }^{4}$ and Richard Gearry ${ }^{5}$ \\ 1 The New Zealand Institute for Plant \& Food Research Limited, Lincoln 7608, New Zealand; \\ sarah.eady@plantandfod.co.nz \\ 2 The New Zealand Institute for Plant \& Food Research Limited, Palmerston North 4410, New Zealand; \\ chrissie.butts@plantandfood.co.nz (C.B.); duncan.hedderley@plantandfood.co.nz (D.H.) \\ 3 Drummond Food Science Advisory Limited, Christchurch 7682, New Zealand; lynley_dfsa@me.com \\ 4 Zespri International Ltd., Mount Manganui 3419, New Zealand; Juliet.Ansell@zespri.com \\ 5 Department of Medicine, University of Otago, Christchurch 8011, New Zealand; \\ Richard.Gearry@cdhb.health.nz \\ * Correspondence: alison.wallace@plantandfood.co.nz; Tel.: +64-274822018 \\ † Presented at the 2019 Annual Meeting of the Nutrition Society of New Zealand, Napier, New Zealand, \\ 28-29 November 2019.
}

Published: 16 December 2019

Functional gastrointestinal disorders including constipation affect up to $14 \%$ of the world's population. Treatment is difficult and challenging resulting in a need for alternative safe and effective therapies. The present study investigated whether daily consumption of three gold-fleshed kiwifruit could alleviate constipation and improve gastrointestinal discomfort in mildly constipated individuals with and without pain. A total of thirty-two participants were enrolled in a 16-week randomised, single-blind, crossover study. Participants received either three 'Zesy002' kiwifruit or $14.75 \mathrm{~g}$ Metamucil ${ }^{\circledR}$ (5 g dietary fibre/d (a positive control)) for 4 weeks each with a 4-week washout between treatments. A 2-week washout period was included at the beginning and end of the study. Daily bowel habit diaries were kept throughout the study. The primary outcome measure was differences in the number of complete spontaneous bowel movements (CSBM). Secondary outcome measures were bowel movement frequency and stool form as well as digestive symptoms and comfort. The number of CSBM per week was significantly greater during daily consumption of three kiwifruit compared with the baseline $(6 \cdot 3 v \cdot 3 \cdot 3 ; p<0.05)$ and the Metamuci ${ }^{\circledR}$ treatment $(6 \cdot 3 v .4 \cdot 5 ; p$ $<0.05)$. Stool consistency was also improved, with kiwifruit producing softer stools and less straining $(p<0.05)$. Gastrointestinal discomfort was also improved compared with baseline for abdominal pain, constipation and indigestion $(p<0.05)$ during the kiwifruit intervention and constipation during the Metamucil $^{\circledast}$ intervention $(p<0.05)$. This randomised controlled trial demonstrates that daily consumption of three gold-fleshed kiwifruit is associated with a significant increase of two CSBM per week and reduction in gastrointestinal discomfort in mildly constipated adults.

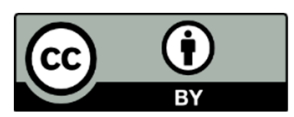

(C) 2019 by the authors. Licensee MDPI, Basel, Switzerland. This article is an open access article distributed under the terms and conditions of the Creative Commons Attribution (CC BY) license (http://creativecommons.org/licenses/by/4.0/). 